\title{
On the prediction of material properties and topology for optimal continuum structures
}

\author{
J.M. Guedes \\ Instituto Superior Tecnico, Av. Rovisco Pais, P-1096 Lisbon, Portugal \\ J.E. Taylor \\ Dept. of Aerospace Engineering, University of Michigan, Ann Arbor, MI 48109, USA
}

\begin{abstract}
A new formulation is presented for mathematical modelling to predict material properties for the optimal design of continuum structures. The method is based on an extended form of an already established characterization for continuum design, where the material properties tensor for an arbitrary structural continuum appears as the design variable. The extension is comprised of means to represent an independently specified unit relative cost factor, which appears simply as a weighting function in the argument of the isoperimetric (cost) constraint of the original model. A procedure is demonstrated where optimal black/white topology is predicted out of a sequence of solutions to material properties design problems having this generalized cost formulation form. A systematic adjustment is made in the unit relative cost field for each subsequent solution step in the sequence, and at the stage identified with final topology, no more than a small fraction of a percent of the total element area in the system has material property density off the bounding "black" or "white" levels. This technique is effective for the prediction of optimal black/white topology design for design around obstacles of arbitrary shape, as well as the more unusual topology design problems. Results are presented for 2D examples of both types of problem. In addition to the treatment for (the usual) minimum compliance design, an alternate formulation of the design problem is presented as well, one that provides for the prediction of optimum topology with a generalized measure of compliance as the objective.
\end{abstract}

\section{Introduction}

Among established characterizations within structural optimization for the determination of continuum topology design, most are modelled on the assumption that the structure is composed of two-phase material, where generally each phase is taken to be locally isotropic. Literature in the general area of topology design for structures, which is concentrated in the recent decade, is summarized very well in Bendsøe's book (1995), in the proceedings by Bendsøe and Mota Soares (1993), and in the survey article by Rozvany et al. (1995). The various alternatives employed to address the inherent illposedness of the two-phase model are aired thoroughly there, and the reader is referred to these and other resources in the literature on topology design for consideration of the details related to existing models and procedures. The recent work of Petersson and Sigmund (1996), which postdates the cited article and books, describes an alternative approach [a narrowing of the design space via imposition of a bound on the derivative of the design function, a device introduced in the context of plate design by Niordson (1983)] in a way that facilitates gaining insight into the development of various existing models for topology design based on the two-phase characterization.

The formulation of this paper is based on a recent development in analytical modelling for the prediction of optimal material properties where, in contrast to the two-phase basis of earlier methods, structural material is represented in the form of an arbitrary (free) material properties tensor field. Using this model, optimal topology is predicted in effect as a part of the determination of optimal fields for the material properties tensor. Specifically, supposing that resulting values for a measure of the material properties tensor are identified with a scale of greys, then a plot of the greys summarizes topologies at the respective grey levels. The purpose here is to show how an extended version of the material properties design formulation may be exploited to form a method for the generation of computer prediction of refined topologies, i.e. to achieve a resolution from grey-scales results to a black/white topology. Following this alternative approach, certain pathologies experienced in connection with computational solution procedures based on the familiar two-phase models are circumvented. This relates to the facts that the free-material design problem is well-posed, i.e. does not lack closure, and that in the present method the simplicity of this model is preserved throughout the computational procedure. To elaborate on the latter point, using our model the optimal black/white topology design is produced without resort to modifications in the original design problem. In other words, steps such as relaxation of the model, the introduction of synthetic penalization procedures, or the imposition of constraints on the design space, common to existing schemes for topology design, do not arise in the present alternative treatment for the problem.

To consider related background material in more detail, the cited model for the design of optimum continuum structures, which predicts as its solution the optimal material properties field from among admissible "free tensor" fields, was first presented by Bendsøe et al. (1994). [This sort of formulation was extended by Bendsøe et al. (1995) to multipurpose design, and it is described for design with nonlinear materials by Bendsøe et al. (1996)]. As note above, such re- 
sults already represent topology design, but only in the sense where topology is associated with fields of "shades of grey"; the grey level corresponds to the scalar variable identifying material property density. We note that the present model belongs to the category of models for optimal design where the density is uniquely identified with a measure of unit strain energy. For present purposes, namely to obtain a modified form of the "free material design" formulation that serves as the basis to solve for optimal black/white topology, means are introduced to represent arbitrary specified unit relative cost over the field of the structure. A general form for representation of unit or local cost fields is described by Taylor and Washabaugh (1995a,b, 1997); applications to truss design are reported by Taylor (1997). Cost is represented there in generalized form by the introduction into the isoperimetric (cost) constraint of a set of weights applied to a complete form of expression for an invariant measure associated with the continuum material, or for the discrete structure with elements of the truss. The representation here for the cost of continuum material is the same except that, instead of it being expressed in general form, the problem is to be treated for a specific choice for the invariant measure of material properties, in fact one appearing in the paper by Bendsøe et al. (1994).

This paper includes a presentation in brief of the model on which the procedure to predict optimal black/white topology design is based, the form of which follows closely the developments reported by Bendsøe et al. (1994) for minimum compliance design using the free material properties tensor as the design variable. That model, modified to reflect nonuniform unit cost over the field of the structure, is then interpreted into an algorithm form for computational treatment of the problem, and graphical results are presented for an example topology design problem. The same means are also exploited to demonstrate a procedure to predict optimal topology where obstacles are present within the admissible space for the structure. Along a different line, an interpretation is given in detail for an alternative model for the prediction of optimal topology, one where the argument of the objective is a generalized measure of structural compliance in place of the usual minimum compliance criterion.

\section{Analytical problem formulation}

Both notation and presentation for the developments described in this section follow those given by Bendsøe et al. (1994), except for modification of the isoperimetric (cost) constraint as noted. The minimum compliance design for a continuum structure occupying region $\Omega$ and composed of material with modulus $E_{i j k \ell}$, and subject to body force $f$ and to boundary traction $t$ on part $\Gamma_{t}$ of the boundary, is stated symbolically as:

$\min _{\tilde{E}, \tilde{u}}\left\{\ell(\tilde{u})=\int_{\Omega} f_{i} \tilde{u}_{i} \mathrm{~d} \Omega+\int_{\Gamma_{t}} t_{i} \tilde{u}_{i} \mathrm{~d} S\right\}$,

subject to

$\int_{\Omega} \tilde{E}_{i j k \ell} \varepsilon_{i j}(\tilde{u}) \varepsilon_{k \ell}(\tilde{v}) \mathrm{d} \Omega-\ell(\tilde{v})=0, \quad \forall \tilde{v} \in K$,
$\tilde{E} \geq 0, \quad \int_{\Omega} \omega \Psi(\tilde{E}) \mathrm{d} \Omega-R \leq 0$.

The symmetries usual for an elasticity modulus tensor apply, and loads $f_{i}$ and $t_{i}$, the designation of $\Omega, \Gamma_{t}$ and $\Gamma_{u}$, unit relative cost function $\omega$, and bound $R$ on total "cost" are data. Set $K$ identifies kinematically admissible vector fields $\tilde{u}, \tilde{v}$, and with $\Gamma$ representing the complete boundary and $\Gamma_{u}$ the fixed part, we require that $\Gamma=\Gamma_{t} \cup \Gamma_{u}$; $\Gamma_{t} \cap \Gamma_{u}=0$. The symbol $\Psi$ stands for an invariant of the modulus tensor, at this point undesignated, $\omega$ and $R$ symbolize, respectively, a (specified) unit relative cost distribution and the specified bound on global cost. Solutions for the material properties (topology) design for different unit relative cost fields are to be compared, eventually, and so admissible cost fields $\omega(x)>0$ are to belong to a normalized set, e.g. $\int_{\Omega} \omega \mathrm{d} V=N ; N>0$ specified (the value $N$ may be varied in computational procedures, in order to maintain constant volume fraction of material). The second constraint in (1) represents that the material tensor is positive semidefinite, i.e. $\tilde{E}_{i j k \ell} \tilde{\varepsilon}_{i j} \varepsilon_{\tilde{k} \ell} \geq 0 ; \tilde{\varepsilon}_{i j}=\tilde{\varepsilon_{j i}} \forall i, j$.

Problem (1) can be stated in the alternative form (Bendsøe et al. 1994)

$$
\left.\max _{\tilde{E} \geq 0}\left\{\min _{\Omega} \Pi \Psi(\tilde{E}) \mathrm{d} \Omega-R \leq 0, \tilde{u}\right)\right\},
$$

where $I I$ represents potential energy associated with the linear elastostatics problem. Noting that the local measure of cost of material is taken to be an invariant of the modulus tensor, i.e. a scalar function in $\Omega$, "maximization with respect to the tensor" may be interpreted separately with respect to local dependence on orientation, and with respect to variation over the field. Accordingly, with the introduction of $\rho$ to represent the latter aspect, problem statement (2) is rewritten as

$$
\begin{aligned}
& \max \\
& 0<\rho_{\min } \leq \rho \leq \rho_{\max } \\
& \int_{\Omega} \omega \rho \mathrm{d} \Omega-R \leq 0
\end{aligned}\left\{\begin{array} { l } 
{ \operatorname { m a x } _ { \tilde { E } \geq 0 } } \\
{ \Psi ( \tilde { E } ) = \rho }
\end{array} \left\{\min _{\tilde{u} \in U} \Pi(\rho, \tilde{E}, \tilde{u}) .\right.\right.
$$

From this point onward, the development is written for the example where the invariant $\Psi$ is identified specifically with the trace of the modulus tensor, $\Psi(\tilde{E})=E_{i j i j}$. The innermost max and min can be interchanged, and the solution for the inner max provides the result for optimal material properties [details are given by Bendsøe et al. (1994)], namely,

$E_{i j k \ell}=\rho \frac{\varepsilon_{i j} \varepsilon_{k \ell}}{\left\|\varepsilon_{i j}\right\|^{2}}$.

With the substitution of this result, the corresponding optimal strain energy density, say $W$, is given by

$W:=\frac{1}{2} E_{i j k \ell} \varepsilon_{i j} \varepsilon_{k \ell}=\frac{1}{2} \rho \varepsilon_{i j} \varepsilon_{i j}$

(which equals the strain energy of an isotropic linearly elastic material). In view of (5), problem statement (3) can now be written more simply as

$$
\begin{aligned}
& \max \\
& 0<\rho_{\min } \leq \rho \leq \rho_{\max } \\
& \int_{\Omega} \omega \rho \mathrm{d} \Omega-R \leq 0
\end{aligned} \min _{\tilde{u} \in U}\left\{\frac{1}{2} \int_{\Omega} \rho \varepsilon_{i j} \varepsilon_{i j} \mathrm{~d} \Omega-\ell(\tilde{u})\right\} .
$$

Thus what remains from (1) is the requirement to predict the optimal distribution $\rho(x)$ and the associated equilibrium 
state $u(x)$. Once the optimal distribution function $\rho$ has been obtained, the optimal pointwise material properties are determined from (4).

We note that the weighted cost constraint introduced in the present formulation has the same form as that of the corresponding isoperimetric constraint in the original problem statement from Bendsøe et al. (1994), so the mathematical structure of the design problem is unchanged from the original one and the solution to (6) is (still) a saddlepoint. [The prior material properties design problem is in fact recovered from the present one for the case $\omega \equiv 1$.] Also, given (5), the optimality condition for the design of optimal material properties may be stated as

$\frac{1}{2} \varepsilon_{i j} \varepsilon_{i j}=\omega \Gamma+\bar{\gamma}-\underline{\gamma}$

where $\Gamma>0, \bar{\gamma}(x) \geq 0$, and $\gamma(x) \geq 0$ represent multipliers on the global and local design constraints. This result is the same as that presented by Bendsøe et al. (1994) except for the presence of unit relative cost factor $\omega$, and as noted there it has the appearance of the optimality condition for design of thickness for an optimal sheet design. Thus by (7) the result in its present form states that the unit strain energy field equals the prescribed weighting function $\omega(x)$ times constant $\Gamma$ in the "design domain", i.e. where $\rho_{\min }<\rho<\rho_{\max }$.

\section{An alternative formulation}

The purpose here is to present a formulation that is generalized somewhat with respect to the objective. Thus while prior problem statements (1) and (2) represent minimization of structural compliance, for the generalized form the objective is taken to be a measure over the structure of the inner product of a specified weighting and the magnitude of displacement. [An alternate form where specific weighted measures of displacement appear as constraints is described by Rozvany and Zhou (1993).] This problem can be expressed conveniently with the introduction of an additional vector field variable, say $\beta_{k}(x) \geq 0$ in $\Omega, k=1,2,3$, which bounds the absolute value of displacement components $u_{k}(x)$ [this provides for the present vector field problem the convenience realized with the introduction of a scalar bound in the interpretation given to minmax problems by Bendsøe and Taylor (1984).] The specified field $q_{k}(x) \geq 0$ in $\Omega, k=1,2,3$, prescribes a weighting in the objective, one which in fact weights $\left|u_{k}(x)\right|$ as is to be confirmed below (the means to include a weighted measure of boundary displacements in the objective is discussed below as well). With the introduction of these quantities, the design problem can be expressed as (tildes used above to identify elements of the admissible sets are omitted here for simplicity)

$\min _{\beta_{k}, u_{k}, v_{k}, \varepsilon_{k \ell}, \hat{\varepsilon}_{k \ell}, E}\left[\int_{\Omega} q_{k} \beta_{k} \mathrm{~d} V\right]$,

such that

$-\beta_{k} \leq 0, \quad u_{k}-\beta_{k} \leq 0, \quad-u_{k}-\beta_{k} \leq 0$,

$\varepsilon_{k \ell}-\frac{1}{2}\left(u_{k, \ell}+u_{\ell, k}\right)=0, \quad \hat{\varepsilon}_{k \ell}-\frac{1}{2}\left(v_{k, \ell}+v_{\ell, k}\right)=0$,

$-E \leq 0$,

$\int_{\Omega} f_{k} v_{k} \mathrm{~d} V+\int_{\Gamma_{i}} t_{k} v_{k}-\int_{\Omega} E_{i j k \ell} \varepsilon_{i j} \hat{\varepsilon}_{k \ell} \mathrm{d} V \leq 0, \quad \forall v \in K$,
$\int_{\Omega} \omega \Psi(E) \mathrm{d} V-R \leq 0$

A version of this formulation applicable for truss design is described by Taylor (1997). We suppose here that the null solution to (8) is excluded; this may be enforced objectively by adding the constraint $Q-\int_{\Omega} E_{i j k \ell} \varepsilon_{i j} \hat{\varepsilon}_{k \ell} \mathrm{d} V \leq 0, Q>0$ in the formulation. To do so would not lead to anything of use in the present interpretation of the model, however, so for the sake of simplicity this concern is not addressed here. Notation in (8) follows that of the previous problem statements, but note the additional $\hat{\varepsilon}_{i j}$ and $v_{k}$ representing "adjoint strain and displacement". Given that the set of admissible $v_{k}$ is defined in the usual way, the seventh constraint in the list assures that $\varepsilon_{i j} ; u_{k}$ equilibrate the prescribed loads. The first three entries in the constraint list simply reflect the stated role of bounding function $\beta_{k}(x)$. Also, with the deformation kinematics requirement expressed explicitly (the fourth and fifth constraints), strains $\varepsilon_{i j}, \hat{\varepsilon}_{i j}$ and displacements $u_{k}, v_{k}$ are variationally independent. Note that while representation of the extremum problem statement for design of the material tensor is in a sense more cumbersome in the extended form (8), its interpretation becomes somewhat simpler to follow in the process. The presence of strain-displacement relations as explicit constraints facilitates extension of the formulation to cover finite strain modelling, for example [this idea appears in a different context in the book by Washizu (1982)].

To proceed with the intepretation of the problem, multipliers associated with the first five constraints in (8), respectively, are designated as $\kappa_{k}, \mu_{k}, \eta_{k}, s_{k \ell}$ and $\hat{s}_{k \ell}$. Then the generalized KKT conditions associated with the first three constraints are stated,

$\kappa_{k} \beta_{k}=0, \quad \kappa_{k} \geq 0, \quad \forall x \in \Omega$, $\mu_{k}\left(u_{k}-\beta_{k}\right)=0, \quad \mu_{k} \geq 0, \quad \forall x \in \Omega$, $\eta_{k}\left(-u_{k}-\beta_{k}\right)=0, \quad \eta_{k} \geq 0, \quad \forall x \in \Omega$.

Stationarity w.r.t. $\beta_{k}(x)$ requires that at the solution

$q_{k}-\left(\kappa_{k}+\mu_{k}+\eta_{k}\right)=0, \quad \forall x \in \Omega$.

Suppose that $\kappa_{k}>0$ in a finite interval of $\Omega$. Then according to $(9) \beta_{k}(x)$ has a value identical to zero in that interval, which requires in turn by the original constraints that the primary displacement $u_{k}(x)$ is identical to zero there. Such "intervalwise rigid body displacement" is inadmissible (see below for confirmation), and so it follows that $\beta_{k}>0 \rightarrow$ $\kappa_{k}=0$ almost everywhere (a.e.) in $\Omega$. The set of points at which $\beta_{k}$ has value zero is designated $\Omega_{o}$. Note also by (10) and (11) that this requires $\mu_{k} \eta_{k}=0$ outside $\Omega_{o}$. As a consequence, the remaining possibilities for meeting (9)-(12) are summarized by

$\mu_{k}=q_{k}, \quad u_{k}=\beta_{k} \quad$ say for $\quad x \in \Omega_{+}$,

$\eta_{k}=q_{k}, \quad u_{k}=-\beta_{k} \quad$ say for $\quad x \in \Omega_{-}$.

With weight $q_{k}$ specified to have positive value throughout $\Omega$, the closure in domain $\Omega_{0} \cup \Omega_{+} \cup \Omega_{-}=\Omega$ follows. Finally, from (13), (14) and the closure result we have that $\left|u_{k}\right|=\beta_{k}$, and this qualifies that the objective stated originally, namely $\int_{\Omega} q_{k}\left|u_{k}\right| \mathrm{d} V$, is indeed properly identified with the argument of "min" in (8).

Conditions for stationarity in problem (8) w.r.t. displacement $u_{k}(x)$ and strain $\varepsilon_{i j}(x)$, and w.r.t. the adjoint displacement and strain $v_{k}(x)$ and $\hat{\varepsilon}_{i j}(x)$ are to be examined next. 
With the introduction of $\hat{s}_{k \ell}$ and $s_{k \ell}$ as multipliers associated with the fourth and fifth constraints in (8), and with the multiplier on the seventh constraint symbolized by $\Lambda$, the cited stationarity conditions are

$\left(\mu_{k}-\eta_{k}\right)+\hat{s}_{k \ell, \ell}=0$ in $\Omega$,

$\hat{s}_{k \ell} n_{\ell}=0$ on $\Gamma$,

$\hat{s}_{k \ell}-\Lambda E_{i j k \ell} \hat{\varepsilon}_{i j}=0$ in $\Omega$,

$\Lambda f_{k}+s_{k \ell, \ell}=0$ in $\Omega$,

$s_{k \ell} \eta_{\ell}-\Lambda t_{k}=0$ on $\Gamma_{t}$,

$s_{k \ell}-\Lambda E_{i j k \ell} \varepsilon_{i j}=0$ in $\Omega$

According to (16) and (18), $\hat{s}_{k \ell} / \Lambda$ and $s_{k \ell} / \Lambda$ measure stress for material $E_{i j k \ell}$ under strains $\hat{\varepsilon}_{k \ell}$ and $\varepsilon_{k \ell}$, respectively. At the same time, (17) and (17b) identify the field $s_{k \ell} / \Lambda$ as the equilibrant of actual loads $p_{k}$ and $t_{k}$. From (15) and (15b) stress field $\hat{s}_{k \ell} / \Lambda$ equilibrates "loads" $\tilde{\mu}_{k}:=\mu_{k} / \Lambda$ and $\bar{\eta}_{k}:=\bar{\eta}_{k} / \Lambda$ or, making use of the result (13) and (14) on the decomposition of $\Omega,(15)$ can be interpreted into the system

$\hat{s}_{k \ell, \ell}+q_{k}=0$ in $\Omega_{+}$,

$\hat{s}_{k \ell, \ell}-q_{k}=0$ in $\Omega$.

Points in $\Omega_{o}$ represent intersections of $\Omega_{+}$and $\Omega_{-}$, i.e. points where $u_{k}$ switches sign. Boundary condition $(15 \mathrm{~b})$ together with (19) and (20) summarize the equilibrium requirement on adjoint stress $\hat{s}_{k \ell} / \Lambda$.

Formulation (8) may be elaborated to provide for the inclusion of the objective of weighted displacement on the boundaries. Supposing that a weighting $b_{k}(x)>0$ on $\Gamma$ is specified, and with the introduction of the (additional) bounding function $\delta_{k}(x)>0$ on $\Gamma$, for the extended problem we seek to predict the solution to

$\min \left[\int_{\Omega} q_{k} \beta_{k} \mathrm{~d} V+\int_{\Omega} b_{k} \delta_{k} \mathrm{~d} S\right]$,

with the additional constraints

$$
\left.\begin{array}{l}
-\delta_{k} \leq 0 \\
u_{k}-\delta_{k} \leq 0 \\
-u_{k}-\delta_{k} \leq 0
\end{array}\right\} \quad \text { on } \Gamma
$$

appended to the original constraint list. Following in form the development given above, interpretation of the necessary conditions for the modified problem provide that $\delta_{k}=\left|u_{k}\right|$ on $\Gamma$ (it follows as well that $\delta_{k}=\beta_{k}$ on $\Gamma$, since $u_{k}$ is continuous in $\Omega \cup \Gamma$ ), and the prior boundary condition (15b) becomes $\hat{s}_{k \ell} \eta_{\ell}-b_{k}=0$ on $\Gamma$.

To summarize, for the problem in its extended form it is possible to design for weighted objective on either interior or boundary displacement alone, or for combined, independently weighted measure of displacement on both $\Omega$ and $\Gamma$. Also note that, assuming the earlier choice for invariant $\Psi(E)$ applies here, the optimality condition for the extended formulation (8) has the same form as (7) for the original minimum compliance problem, but is expressed in terms of a measure of unit mutual energy, i.e.

$\Lambda \varepsilon_{i j} \hat{\varepsilon}_{i j}=\omega \Gamma+\bar{\gamma}-\underline{\gamma}$

[the terminology "mutual" energy was used, for example, by Prager (1972)]. While this result matches in simplicity its counterpart for the conventional minimum compliance problem, means to identify the optimal material tensor are not readily available in the case of the generalized problem. Of course, in the case that the weighting functions $q_{k}$ and $b_{k}$ are equal to actual body force and boundary loads, the present system reduces to one equivalent to that of the prior section.

We remark that the generalized compliance formulation (8) can be extended directly to accommodate nonlinear deformation kinematics, simply. via an upgrade of the fourth and fifth constraints there to represent the Green strain measure in place of the linear one [the fimite strain problem was treated e.g. by Guedes and Taylor (1996)]. (a)

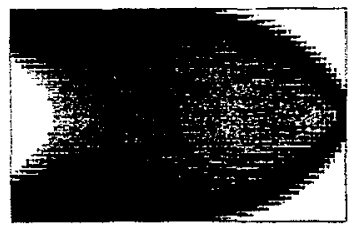

(b)

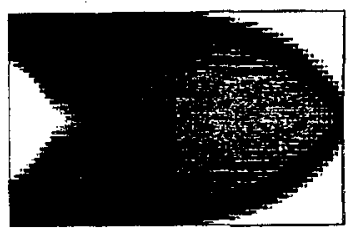

(c)

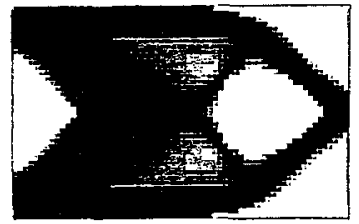

(d)

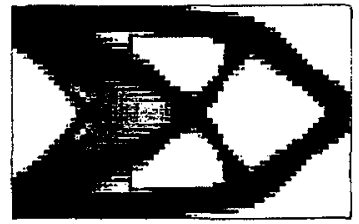

(e)

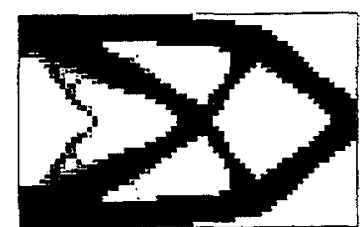

(f)

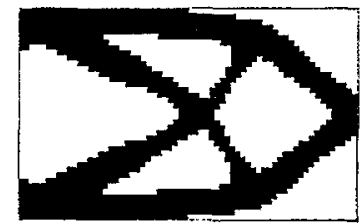

Fig. 1. Sequence from design (a) for uniform cost to the blackwhite optimal design (f) -solutions obtained using 8-node rectangular elements and with rapid stepwise adjustment in the unit cost function

\section{Computational solution}

Optimal topology design is predicted using a cyclic procedure, where the result at each cyclic step is a solution to (3) corresponding to a specific designation for the unit relative cost $\omega(x)$. The method used to obtain the results presented by Bendsøe et al. (1994) is followed here to produce these stepwise solutions, namely an optimality based approach coupled with a conventional finite element model solver. As was noted in the cited paper, this is a technique that has been used over the past two decades to obtain numerical results for a wide range of structural optimization problems. The present procedure to determine optimal topology is initiated by solving for the optimal distribution $\rho$ corresponding to uniform unit relative cost over the entire structure. The unit relative cost field is adjusted at each subsequent step as follows. A threshold or cutoff value of the material property density $\rho$ is designated, and a uniform, higher value is given to the unit relative cost over the areas where density has a value below the threshold value. The solution is obtained for optimal $\rho(x)$ corresponding to this new unit cost field to 
(a)

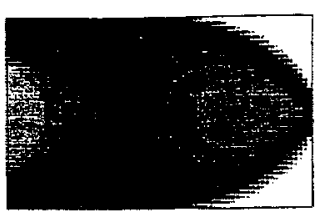

(e)

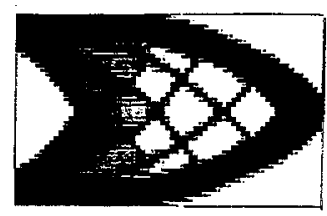

(b)

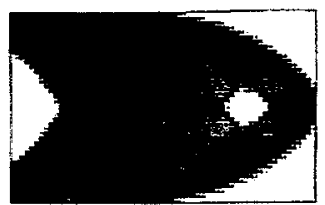

(c)

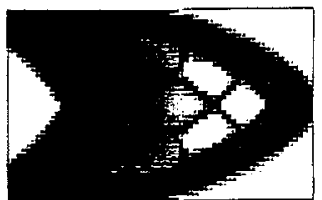

(d)

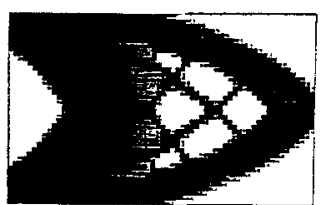

(f)

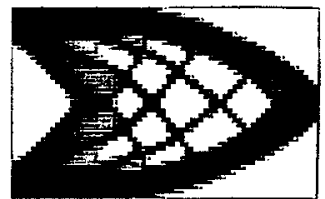

(g)

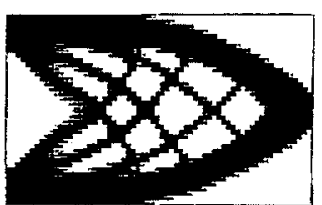

(h)

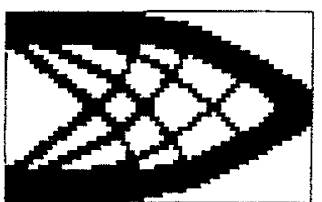

(a)

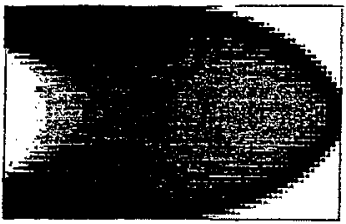

(b)

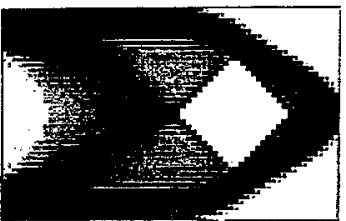

(c)

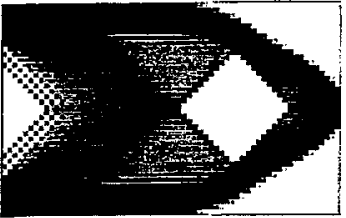

Fig. 2. Sequence fro the example of Fig. 1, but where design results (a) through (h) are obtained with gradual adjustment of the unit cost field

(a)

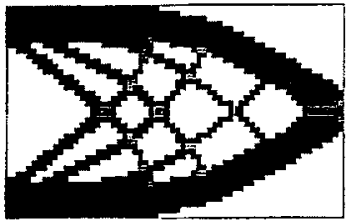

(b)

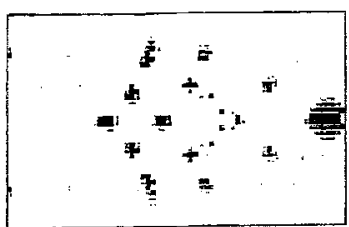

(c)

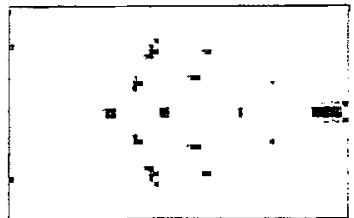

(d)

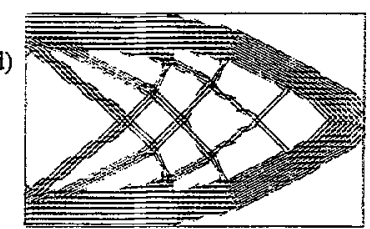

Fig. 3. Interpretation of the solution design (h) of Fig. 2: (a) through (c) show respectively the 1111, 1122 and 2222 components of the material modulus tensor; the magnitude and direction of principle strains are given in (d). The scale shown for "shades of grey" applies to all examples

complete the step. Results for each such step in the method reflect a relative increase on concentration of material density, evolving toward the value for the upper bound on $\rho$ in the regions remaining at lower unit relative cost. The evolution from an initially broad spectrum of shades of grey toward a discrete black/white design is described in Figs. 1, 2, 4 and 5 for the numerical examples. We note that the (relatively unrefined) solution process is carried out in a "user interactive mode", and the rates for adjustment of the "threshold value for density" and the "increased unit relative cost" are at the

(a)

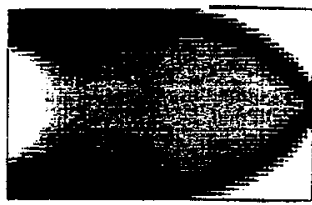

(b)

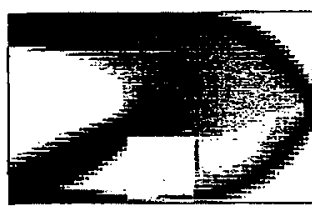

(c)

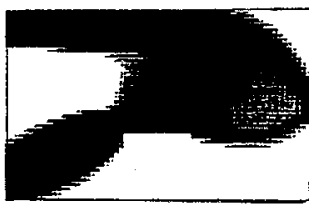

(d)

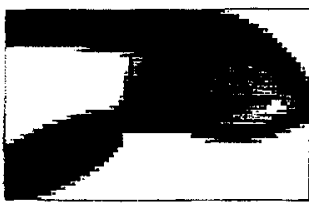

(d)

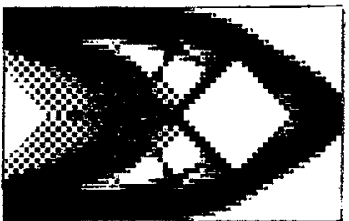

(e)

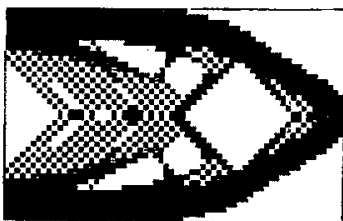

Fig. 4. Sequence (a) through (f) for the evolution to black-white topology with a required hole or obstacle within the region of the original result; checkerboarding is attributed to the low-order element (4-node rectangular) used here

(f)

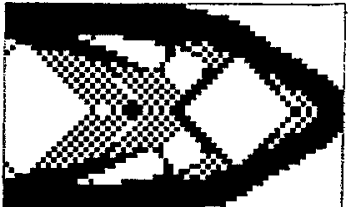

(e)

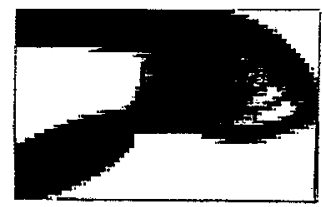

(f)

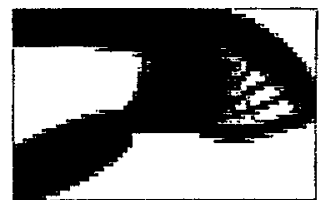

(g)

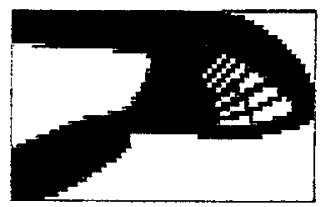

(h)

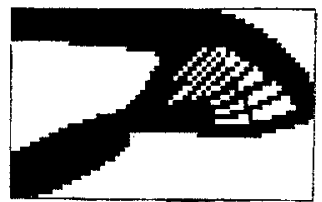

Fig. 5. Evolution for an example similar to that of Fig. 4, but where the hole or obstacle is placed along the edge of the "design space"; results were obtained using 8-node elements

user's discretion. While the overall computational procedure has not been subject to study and testing (our purpose here is just to provide examples of its workings), it appears that 
on the order of fifteen steps in the cyclic procedure suffices to produce high quality renditions of optimal black/white topology.

All the examples treated here relate to the design of a 2D continuum model of an end loaded cantilevered beam. The structure is supported along its left edge and loaded by a local distribution centred around mid-height on the right edge. All computations are performed using four- or eightnode rectangular elements on a 40 by 64 element mesh, and the objective in all cases is to minimize (conventional) compliance. Sketches shown in Figs. 1a-f reflect the evolution of "material property density" distributions, from the initial step described above and shown in Fig. 1a, through several intermediate solutions (Figs. 1c-e), to our final result of Fig. 1f. (Values of "density" corresponding to shades of grey are indicated by the scale shown in Fig. 3 ; the same scale applies to all examples). As a measure of convergence toward a pure black/white design, we note that in the solution corresponding to Fig. 1f, only two out of the total number of elements in the structure have density between the upper and lower limit values. Note that the relative "white-to-black" density for these results has the order of magnitude $10^{7}$. Results shown in Fig. 2 are for the same example problem, but a significantly more gradual rendition of topology for this example reflects finer structural detail, the scale of detail in fact becomes set early in the evolution process for both sets of computation. Diagrams in Fig. 3 provide information on the distributions of the components of the modulus tensor, and on the orientation and magnitude of principal strains. A comparison of Figs. 3a and b suggests that the 1122 component of the material tensor enters the optimal result, at the expense of the 1111 component, in areas where the member-like regions intersect. This reflects the relatively more complex stressstate in such areas, which in fact act as "joints". For the remaining two examples, a part of the original design space is to be left open in the final optimal designs, as would be the case where a hole is required or an obstacle is present in the "design space". In each case, the "hole" is produced by the imposition of relatively high unit cost in the area designated to be void of structure, i.e. by the same means as those exploited to obtain the black-white topology in the overall structure. Computational results for these two problems are shown in Figs. 4 and 5; the problem settings are explained in the figure captions.

The approach used here to obtain numerical results clearly depends on the management of the cyclic procedure, and our limited experience does not support the drawing of detailed conclusions. We observe that the computational procedure converges dependably and, as was indicated above, according to present experience on the order of 15 solution steps suffices to produce a refined final design result. [This compares favourably with projected costs of the "slope constrained method (Petersson and Sigmund 1996)"], for example, where prediction of a final design result is estimated to require from 100 to 1000 solutions of the original problem, i.e. the one without slope constraints.

\section{Summary}

A method has been presented for the prediction of topology design for minimum compliance continuum structures. Com- putational examples are presented in $2 \mathrm{D}$, but the model and method are applicable as given for 3D design problems. Results are shown for design around an obstacle as well as for the simple minimum compliance design. The problem is formulated in a way to predict the optimal material properties together with topology design. In this presentation the set of admissible materials includes all possible constitutions for the (realizable, linearly elastic) continuum, and thus the results are distinct compared to those predicted out of more familiar methods for the design of topology. The latter reflect the limitations inherent with the assumption of a two-phase, locally isotropic material for the design. The present technique might be interpreted as a form of filtering, where the originally opaque <shades of grey> design is rendered through the solution process into a "high-resolution" version of design (which is in fact what is meant by the label "topology design" for continuum structures!). The distinctiveness of the present results lies in part with the fact that the continuously varying optimal material properties are predicted along with the "high resolution" definition of structural shape.

In addition to the treatment of the conventional minimum compliance problem, a formulation is presented for design where the integral of an independently weighted measure of displacement is minimized. This alternate formulation provides for the user to designate, in the setting of problems for optimal topology design, where over the structure the (relative) displacement is to be controlled. While this model has not yet been implemented computationally for continuum design, its counterpart expressed for the design of discrete structures has been applied successfully to predict optimal truss designs (Taylor 1997).

In terms of its mathematical structure the extension of the problem formulation from the original "material properties design" one to the form presented here for topology design is notably inconsequential, i.e. the structure of the model is unchanged. However, clearly the form elaborated to account for specified local unit relative cost is significant on practical grounds, as it comprises the basis for what proves to be an efficient and simple technique to predict optimal topology. The efficiency derives largely from the fact that the model which serves as a basis for prediction of topology retains the simplicity (linearity) of the original "material tensor design" problem.

One might anticipate useful future developments to establish a similar approach for applications to nonlinear problems [such purposes may be served by recent developments (Taylor 1996) in variational modelling for nonlinear elasticity], for example, or to predict optimal multipurpose design, and so on. Also, with regard to problem-solving capability, exploitation of the larger potential of the present technique for prediction of optimal topology and material properties design clearly presents a challenge for the creation of more broadly applicable means for computational treatment. It should be of interest in this respect to consider refinements among optimality criterion based methods (see e.g. Zhou and Rozvany 1993) for their potential usefulness.

\section{Acknowledgements}

The work reported here has received support (JET) under AFOSR grant \#F49620-94-1-0442 and from a grant issued by Ford Mo- 
tor Company, Scientific Research Laboratories, and (JMG) from JNICT Project \#PBIC/C/TPR/77404/95 - "Determinacao De Microstruturas Optimas E Fabrico Rapido De Materias Celulares". The authors appreciate constructive suggestions offered by the reviewers.

\section{References}

Bendsøe, M.P. 1995: Optimization of structural topology, shape and material. Berlin, Heidelberg, New York: Springer

Bendsøe, M.P.; Mota Soares, C.A. (eds.) 1993: Topology design of structures. Dordrecht: Kluwer

Bendsøe, M.P.; Taylor, J.E. 1984: An interpretation for min-max structural design problems including a method for relaxing constraints. Int. J. Solids 6 Struct. 20,310-314

Bendsøe, M.P.; Diaz, A.; Lipton, R.; Taylor, J.E. 1995: Optimal design of material properties and material distribution for multiple loading conditions. Int. J. Num. Meth. Engrg. 38, 1149-1170

Bendsøe, M.P.; Guedes, J.M.; Haber, R.B.; Pedersen, P.; Taylor, J.E. 1994: An analytical model to predict optimal material properties in the context of optimal structural design. J. Appl. Mech. 61, 930-937

Bendsøe, M.P.; Guedes, J.M.; Plaxton, S.; Taylor, J.E. 1996: Optimization of structure and material properties for solids composed of softening material. Int. J. Solids $\mathcal{E}$ Struct. 33, 1799-1813

Guedes, J.M.; Taylor, J.E. 1996: A formulation for generalized hyperelasticity using a composition of potentials. (Publication pending)

Niordson, F.I. 1983: Optimal design of plates with a constraint on the slope of the thickness function. Int. J. Solids 6 Struct. 19, $141-151$

Petersson, J.; Sigmund, O. 1996: Slope constrained topology optimization. (Publication pending)
Prager, W, 1972: Conditions for structural optimailty, Comp. E Struct. 1, 833-840

Rozvany, G.I.N.; Bendsøe, M.P.; Kirsch, U. 1995: Layout optimization of structures. Appl. Mech. Rev. 48, 41-119

Rozvany, G.I.N.; Zhou, M. 1993: Continuum-based optimality criteria (COC) methods: An introduction. In: Rozvany, G.I.N. (ed.) Optimization of large structural systems (Proc. NATO/DFG ASI, held in Berchtesgaden, Germany, 1991), pp. 1-26. Dordrecht: Kluwer

Taylor, J.E. 1996: Generalized potential formulations for elastostatics of constitutively nonlinear structures under general loading. Int. J. Mech. Sci. 39, 537-548

Taylor, J.E. 1997: On the prediction of topology and local properties for optimal truss design. Struct. Optim. 14, 53-62

Taylor, J.E.; Washabaugh, P.D. 1995a: A generalized expression of cost for prediction of the optimal material properties tensor. In: Marques, M.D.P.M.; Rodrigues, J.F. (eds.) Trends in application of mathematics to mechanics. Essex: Longman

Taylor, J.E.; Washabaugh, P.D. 1995b: A generalized expression of cost for prediction of the optimal material properties tensor. In: Proceedings summaries, ASME Summer Mechanics Meeting (held at UCLA)

Taylor, J.E.; Washabaugh, P.D. 1997: On structural optimization formulations with generalized cost constraints. In: Godoy, L.; Rysz, M.; Suarez, L. (eds.) Proc. PACAM V (held in San Juan, Puerto Rico). University of Puerto Rico at Mayaguez

Washizu, K. 1982: Variational methods in elasticity and plasticity. Oxford: Pergamon Press

Zhou, M.; Rozvany, G.I.N 1993: Iterative COC methods - Part I. Iterative methods based on continuum formulation. In: Rozvany, G.I.N. (ed.) Optimization of large structural systems (Proc. NATO/DFG ASI, held in Berchtesgaden, Germany, 1991), pp. 27-58. Dordrecht: Kluwer 